\title{
Electrospinning of Gelatin Nanofiber for Biomedical Application
}

\author{
L. MALEKNIA* and Z. REZAZADEH MAJDI ${ }^{1}$ \\ Department of Textile Engineering, Islamic Azad University, South Tehran Branch, \\ Ahang Street,Nabard Blv, Tehran, Iran. \\ ${ }^{*}$ Corresponding author E-mail: Melika02@ Azad.ac.ir \\ http://dx.doi.org/10.13005/ojc/300470
}

(Received: September 26, 2014; Accepted: November 03, 2014)

\begin{abstract}
A series of new and commercially available polymers have been proposed as matrices for cell regeneration and proliferation for tissue engineering applications but lot of questions like interaction of scaffolds with biological systems, toxicity, in vivo studies etc. have to be thoroughly investigated before the technology can be used for any real practical biomedical application .In this Study gelatin nanofibers were prepared via electrospinning using aqueous solutions of formic acid/water as the solvent instead of cylotoxic solvents. The parameters, such as electric field, concentration of solutions and spinning distance were examined for studying the effects on electrospinability and morphology of gelatin nanofibers mats.Gelatin solutions with various concentrations of gelatin $(20 \%-30 \%$, w/v) were prepared using concentrated aqueous formic $\mathrm{acid} /$ water with ratios $(100 / 0,75 / 25,50 / 50$ and75/25, \%v/v) as solvent at room temperature.Morphology of nanofibres was examined by scanning electron microscopy and diameter, diameter distribution and uniformity were measured with image analyzer software. The lowest diameter of gelatin nanofibers $(197 \mathrm{~nm})$ was achieved when the gelatin concentration was $20 \%$. The results showed that the spinability of gelatin strongly depend on the solvent used.
\end{abstract}

Key words: Gelatin-Acid Formic-Nano Fiber-Electrospinning.

\section{INTRODUCTION}

The electrospinning technique provides non-woven to the order of few nanometers with large surface areas, ease of fictionalization for various purposes and superior mechanical properties. Also, the possibility of large scale productions combined with the simplicity of the process makes this technique very attractive for many different applications. Biomedical field is one of the important application areas among others utilizing the technique of electrospinning like filtration and protective material, electrical and optical applications, sensors, nanofiber reinforced composites etc[Min et al., 2004; Sangamesh et al., 2008]. The importance of electrospinning, in 
general, for biomedical applications like tissue engineering drug release, wound dressing, enzyme immobilization etc(Chen et al., 2007).

Numerous polymer biomaterials have been employed for such medical purposes because they have acceptable biocompatibility, biodegradability and absorbability (Wan et al., 2006). These bio-absorbable polymer materials can act as drug carriers by controlling the release rate of the drug initially loaded in application for drugdelivery systems(Ha et al., 2005). Recently, much attention has been given to research of the blends of the biodegradable polymers, a class of the most attractive biomaterials in view of their good biocompatibility, low cost, high performance and lightweight materials(Eberhart et al., 2003; Peng et al., 1996; Liao et al., 2006).

Gelatin is such a kind of biocompatible and biodegradable material having potential environmental and biomedical applications, where biodegradability and safe elimination of the polymer are required(Ikada et al., 2003; Aou et al., 2006; Xu et al., 2005). Gelatin, which is derived from collagen, has been widely used in clinics for wound dressings, pharmaceuticals and adhesives owing to its biocompatibility, biodegradability and hydrogel characteristics, as well as its formability and cost efficiency(Li et al., 2006). However, unlike synthetic polymers which are generally nonionic, gelatin is a kind of polyelectrolyte polymer, which possesses quite a number of ionizable groups. In addition, the strong hydrogen bonding results in a 3-D macromolecular network, which makes the mobility of the gelatin chains decrease tremendously. So the electrospinnability of gelatin is poor(Huang et al., 2004; Ki et al., 2005). 2,2,2-trifluoroethanol (TFE) was proven to be a suitable solvent for electrospinning of gelatin by Huang et al(Huang et al., 2004). The solutions with concentration between 5 wt. $\%$ and 12.5 wt.\% were successfully electrospun into nanofibres. Ultra-fine fibrous structure without any beads was obtained from 7.5 wt.\% solution. The non-woven mat exhibited the largest tensile modulus and ultimate tensile strength. Gelatin nanofibres were successfully prepared by electrospinning using gelatin/formic acid and gelatin/acetic acid solutions by $\mathrm{Ki}$ and Songchotikunpan et al (Songchotikunpan et al.,
2008; Kim et al., 2008). The objective of this study is to develop a novel solution for electrospinning to prepare gelatin nanofibres. We used of formic acid/ water solvent without addition of any other solvents to prepare gelatin nanofibres. Effects of concentration of formic acid, water and gelatin solutions, electric field and spinning distance on the electrospinnability and morphology were examined.

\section{MATERIALS AND METHODS}

\section{Chemical reagents}

Gelatin and formic acid were supplied by Merk

\section{Methods}

Gelatin solutions with various concentrations of gelatin $(20 \%-30 \%$, w/v) were prepared using concentrated aqueous formic acid/ water with ratios (100/0, 75/25, 50/50 and75/25, \%v/ v) as solvent at room temperature. For electrospinning, each solution was loaded in a $5 \mathrm{ml}$ syringe with a metal capillary attached. The circular orifice of the capillary has an inner diameter of 0.5 $\mathrm{mm}$. A counter electrode was located at about 9-13 $\mathrm{cm}$ apart from the capillary tip. A pressure was applied to the solution to maintain a steady flow rate. The applied voltage was in the range of 12-22 $\mathrm{kV}$. All experiments were conducted at room temperature.

Morphology of nanofibres was examined by scanning electron microscopy (SEM) (Philips XL30, Netherlands) at an accelerating voltage of $20 \mathrm{kV}$. Prior to observation, the samples were coated with gold. The diameter, diameter distribution and uniformity were measured with image analyzer software (microstructure).

\section{RESULTSAND DISCUSSION}

\section{Effect of gelatin concentration}

In the electrospinning of polymer with a certain molecular weight, fibers structure can be stabilized only above a critical concentration and below process limiting viscosity. At room temperature, the gelatin solutions of $12 \%(\mathrm{w} / \mathrm{v})$ concentration and above turned into gel, so they could not be electrospun [refrence] but we prepared 

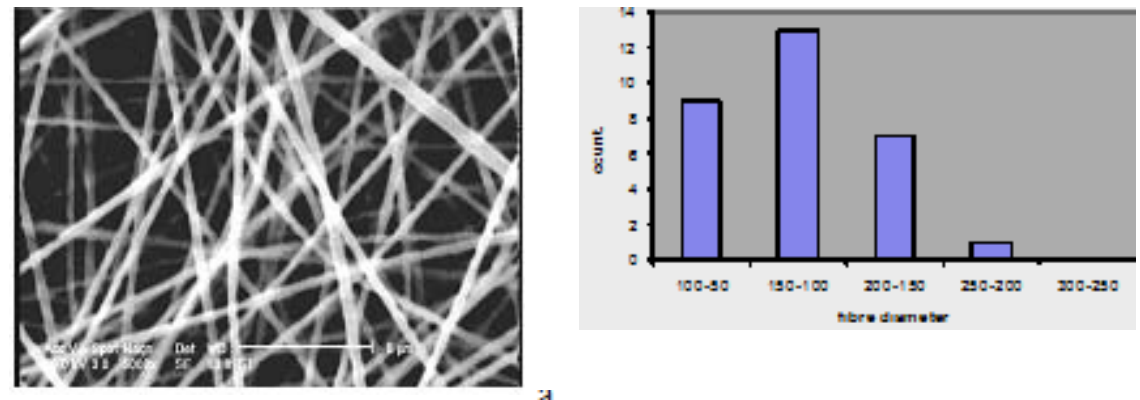

d.
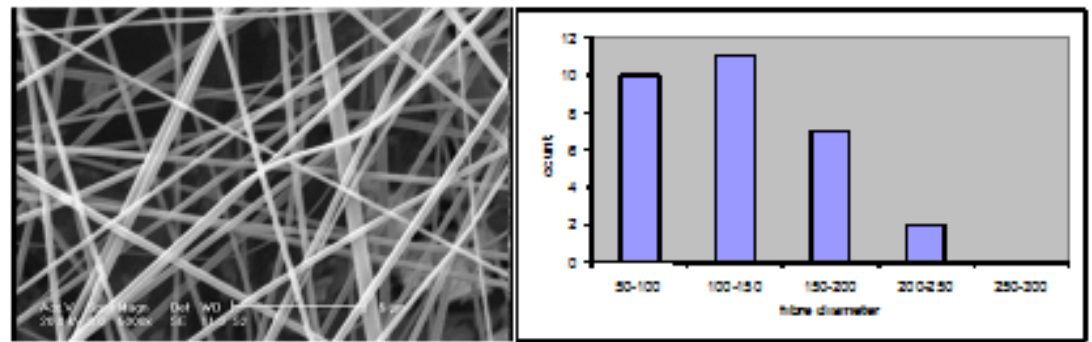

b.

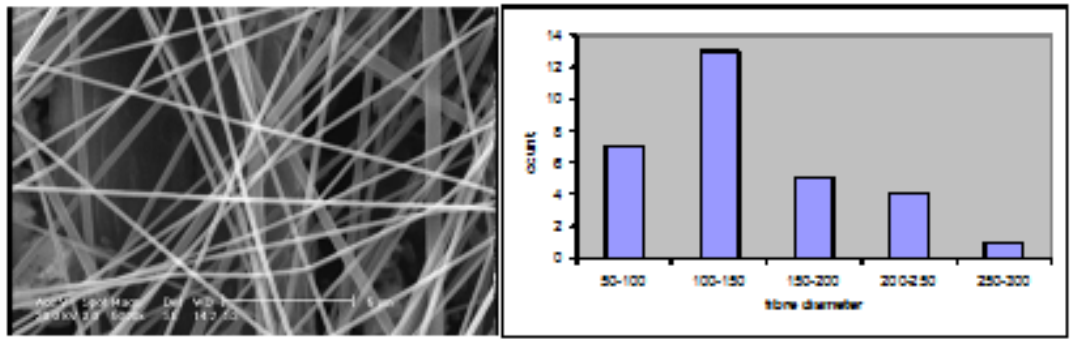

c.

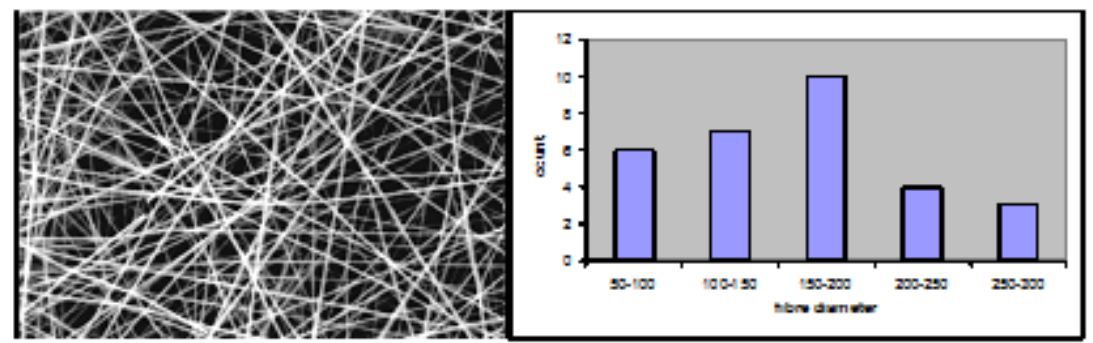

d.
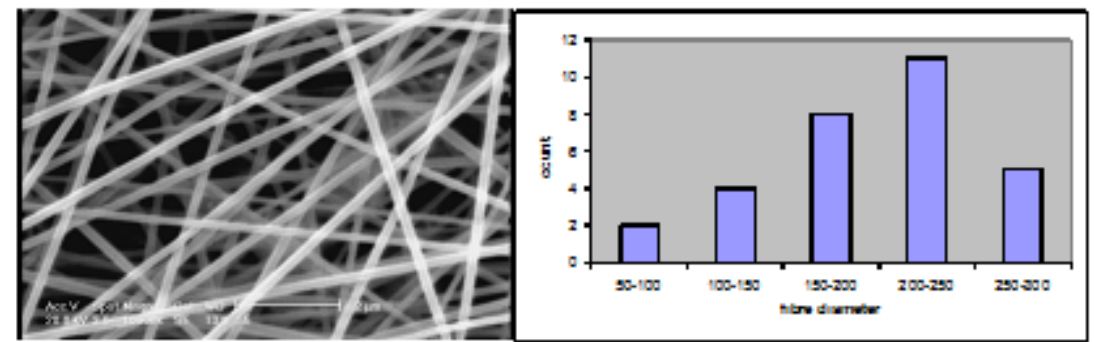

e.

Fig. 1:The SEM micrographs of gelatin nanofibers at various gelatin concentrations,a: $20 \%, \mathrm{~b}: 22.5 \%, \mathrm{c:}: 25 \%, \mathrm{~d}: 27 \%$ and e:30\% respectivly 


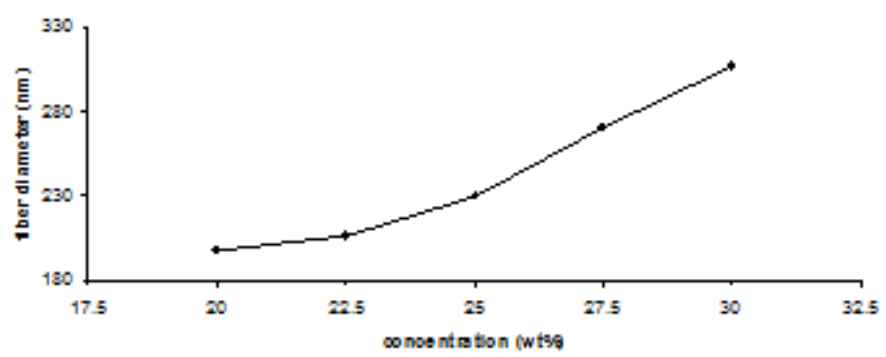

Fig. 2: The average fiber diameter

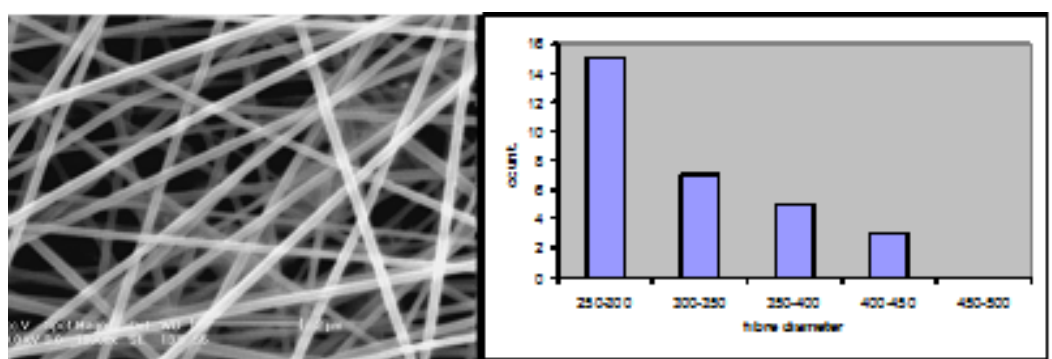

a.

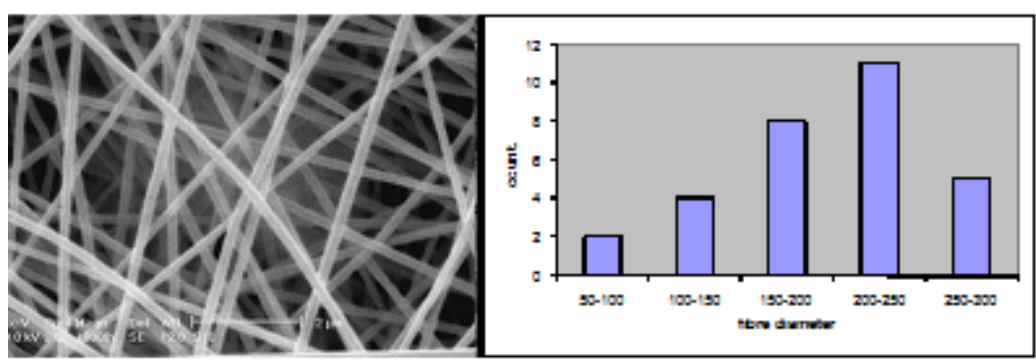

b.
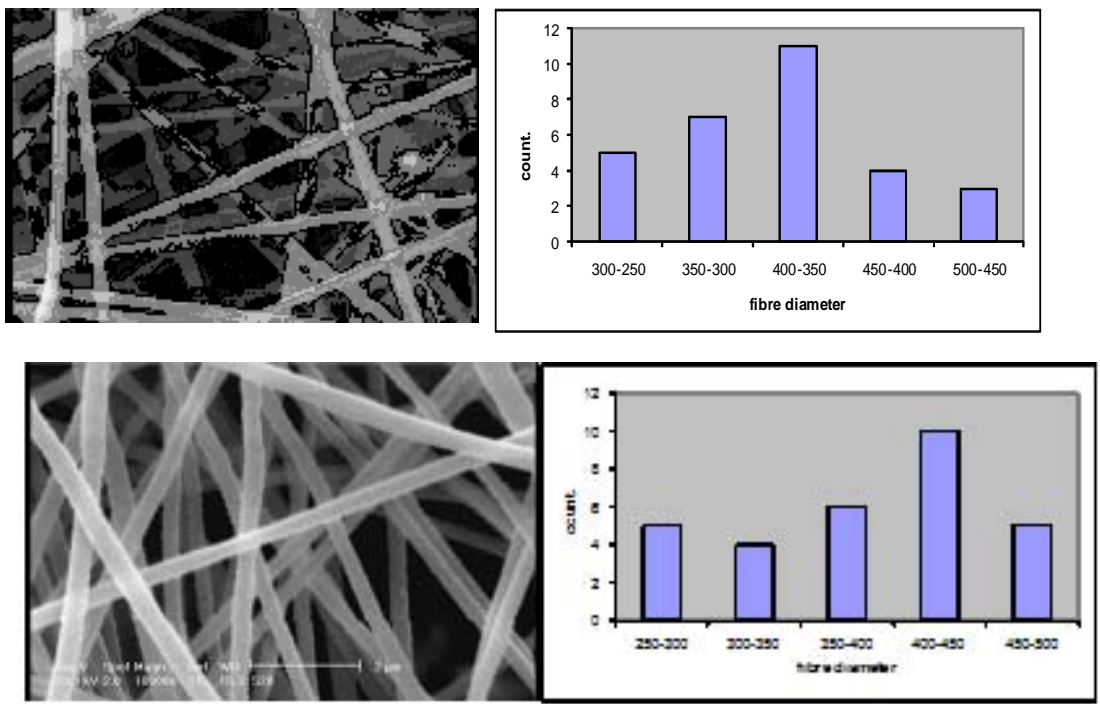

Fig. 3: SEM micrograph and diameter of nanofibers with various acid concentrations, a. $100 \%$, b. $75 \%$, c. $50 \%$ and d. $25 \%$ respectivly 
gelatin nanofibers from $20 \%, 22.5 \%, 25 \%, 27.5 \%$ and $30 \%$ gelatin concentration in formic acid as solvent at room temperature. Beads-on-string structures were observed in electrospun fibers at lower concentration of $20 \%$. The formation of beads can be related to the formation of droplets in electrospray. As at low viscosity, when a high electrical force is applied, formation of droplets occurs because surface tension had a greater effect than the viscoelastic force. With increase of gelatin concentration, bead formation was minimized until smooth fibers were formed at concentration $20 \%$. The change from beads-on-string structures to smooth fibers can probably be attributed to a competition between surface tension and viscosity. Figure 1 shown that the morphology of gelatin nanofibers at (20\%-30\%) of gelatin concentrations. On the other hand, gelatin concentration had a significant effect on the average fiber diameter as shown in Figer 2. The average fiber diameter increased from197 $\mathrm{nm}$ to $307 \mathrm{~nm}$ when the gelatin Concentration was increased from $20 \%$ to $30 \%$. The reason for the positive correlation is that the higher viscosity resisted the extension of the jet.

\section{Effect of Formic acid concentration}

To investigate the effect of formic acid concentration and water as a co solvent on the electrospinnability of gelatin solutions, $30 \%$ gelatin solutions of aqueous formic acid with various acid concentrations, such as $100 \%, 75 \%, 50 \%$ and $25 \%$, were electrospun. It can be seen that when the concentration of formic acid was increased, uniform nanofibres were obtained (Figure 3 ) because the morphology of electrospun nanofibres is also affected by the charge density on the surface of ejected jet, and the higher charge density carried by the jet, the smoother fibers obtained, due to the stronger jet whipping instability ${ }^{30}$. With the increasing of formic acid concentration, the charge density of the solution increased. Therefore uniform fibers were obtained. This picture shown that the average diameter of nanofibers reduce with increased the concentration of formic acid too.

\section{CONCLUSION}

The important advantages of electrospinning technique are the production of very thin fibers with large surface areas, ease of functionalisation for various purposes, superior mechanical properties and ease of process. These advantages provide a wide range of opportunities for their use in many different biomedical applications. These applications range from tissue engineering, drug release, implants, biotransformations to wound healing. Electrospinning assembly can be varied in different ways for combining materials properties with different morphological structures for these applications. Also, although there is ever increasing literature on use of electrospinning for various biomedical applications but still the field is in its infancy. Although a series of new and commercially available polymers have been proposed as matrices for cell regeneration and proliferation for tissue engineering applications but lot of questions like interaction of scaffolds with biological systems, toxicity, in vivo studies etc. have to be thoroughly investigated before the technology can be used for any real practical biomedical application. In this study gelatin nanofibers were prepared via electrospinning using aqueous solutions of formic acid/water as the solvent instead of cylotoxic solvents. The lowest diameter of gelatin nanofibers (197 $\mathrm{nm}$ ) was achieved when the gelatin concentration was $20 \%$. The results showed that the spinability of gelatin strongly depend on the solvent used.

\section{REFERENCES}

1. Min B.M., Lee G., Kim S.H., Nam Y.S., Lee T.S. and Park. W.H, Electrospinning of silk fibroin nanofibers and its effect on the adhesion and spreading of normal human keratinocytes and fibroblasts in vitro Biomaterials, 2004, 25 (7-8), 1289-1297.

2. Sangamesh G.K., Syam P.N., Roshan J.,
Hogan M.V. and Laurencin C.T., Recent Patents Biomed Eng, 2008 , 1; 68-78.

3. Chen C., Gang Lv.,Pan C., Song M.,Wu C.,Guo D.,Wang X., Chen B. and ,Gu ., Biomed. Mater, 2007; 2, L1-L4.

4. Wan Y., Fang Y., Hu Z. and Wu Q., Macromol. Rapid Commun. 2006, 27, 948-54 
5. Ha C.S. and Gardella J. A Surface Chemistry of Biodegradable Polymers for Drug Delivery Systems, Chem. Rev, 2005, 105, 4205-32

6. Eberhart R.C., Su S.H., Nguyen K. T., Zilberman M., Tang L.,Nelson K. D. and Frenkel. P Review: Bioresorbable polymeric stents: current status and future promise, $\mathrm{J}$. Biomater. Sci. Polym. Ed, 2003, 14, 299312.

7. Peng T., Gibula P., Yao K. D. and Goosen M. F. A. Role of polymers in improving the results of stenting in coronary arteries, Biomaterials, 1996, 17, 685-94

8. Liao S.S., Cui F.Z., Zhang W. and Feng Q. L., J. Biomed.Mater. Res. B: Appl. Biomater, 2004, 69, 158-165

9. Ikada Y. and Tsuji T. , Macromol. Rapid Commun, 2000, 21, 117-32

10. Aou K. and Hsu S. L., Macromolecules, 2006, 39, 3337-3344

11. Xu Q., Pang M., Peng Q., Jiang Y., Li J., Wang H. and Zhu M. Effect of different experimental conditions on biodegradable polylactide membranes prepared with supercritical $\mathrm{CO} 2$ as nonsolvent, J. Appl. Polym. Sci 2005, 98, 831-837

12. Li M., Guo Y., Wei Y., MacDiarmid A.G.and Lelkes P.I. Electrospinning polyanilinecontained gelatin nanofibers for tissue engineering applications, Biomaterials, 2006, 27, 2705.

13. Huang Z.M., Zhang Y.Z., Ramakrishna S.and Lim C.T. , Polymer, 2004, 45, 5361.

14. Ki C.S., Baek D.H., Gang K.D., Lee K.H.and Park Y.H. , Polymer, 2005, 46, 5094.

15. Songchotikunpan P., Tattiyakul J.and Supaphol P. Extraction and electrospinning of gelatin from fish skin, Int. J. Biol. Macromol, 2008, 42,247.

16. Kim H.W., Yu H.S.and Lee H.H. Nanofibrous matrices of poly(lactic acid) and gelatin polymeric blends for the improvement of cellular responses, J. Biomed. Mater. Res A, 2008, 87, 25. 